\title{
Intervenções Grupais para Mulheres com Câncer de Mama: Desafios e Possibilidades*
}

\author{
Manoel Antônio dos Santos ${ }^{* *} \&$ Carolina de Souza \\ Universidade de São Paulo, São Paulo, SP, Brasil
}

\begin{abstract}
RESUMO - Este estudo teve por objetivo analisar a produção científica nacional e internacional sobre os grupos de reabilitação psicossocial para mulheres com câncer de mama. O levantamento bibliográfico foi realizado a partir das bases indexadoras LILACS, PubMed, PsycINFO e CINAHL. Foram selecionados 28 artigos, publicados no período de 2000 a 2015, que constituíram o corpus do estudo. Os achados foram agrupados por meio de análise de conteúdo. Os resultados evidenciaram que os grupos de apoio podem resultar em benefícios psicossociais, como redução de estresse, incremento da qualidade de vida, melhora do humor e compartilhamento de informações sobre as diversas opções de tratamentos disponíveis. Conclui-se que participar de grupos de apoio está associado a melhores desfechos psicológicos, contudo, não há evidências que comprovem aumento na expectativa de vida das mulheres.
\end{abstract}

PALAVRAS-CHAVE: neoplasias mamárias, grupos de apoio, psicoterapia de grupo, revisão de literatura

\section{Group Interventions for Women with Breast Cancer: Challenges and Possibilities}

\begin{abstract}
This study aimed to analyze the national and international scientific literature on the topic of psychosocial rehabilitation groups for women with breast cancer. The literature review was conducted from LILACS, PubMed, PsycINFO and CINAHL database. 28 articles published in the period 2000-2015, which constituted the corpus of the study, were selected. The results showed that, in most cases, support groups bring psychosocial benefits, stress reduction, increased and quality of life, mood enhancement and sharing information about different options of treatment available for breast cancer. We conclude that participating in support groups is associated with better psychological outcomes, however, there is no evidence to support an increase in life expectancy of women.
\end{abstract}

KEYWORDS: breast neoplasms, support groups, group psychotherapy, literature review

Segundo tipo de neoplasia mais frequente no mundo, o câncer de mama é o mais comum entre as mulheres no mundo e no Brasil, respondendo por cerca de $25 \%$ dos casos novos a cada ano. Se diagnosticado e tratado oportunamente, o prognóstico é relativamente bom (Brasil, 2017a). Segundo o Instituto Nacional de Câncer José Alencar Gomes da Silva (INCA), investir em novos conhecimentos sobre essa doença é necessário para estabelecer prioridades e alocar recursos para modificar o cenário observado na população brasileira. Em 2016, eram esperados 57.960 casos novos de câncer de mama no país, com 14388 mortes - 181 homens e 14206 mulheres, com um risco estimado de 56,20 casos a cada 100 mil mulheres (Brasil, 2017b).

Com o avanço nos tratamentos oncológicos, propiciados pelo desenvolvimento científico e tecnológico da ciência médica, a sobrevida das pacientes com câncer de mama tem aumentado de forma apreciável (Silva \& Santos, 2010). Por essa razão, nos últimos anos tem crescido a preocupação com as dimensões psicossociais do câncer mamário (Ambrósio \& Santos, 2011; Santos, Ford, Santos, \& Vieira, 2014; Vieira, Santos, Santos, \& Giami, 2014). Há crescente necessidade de compreender os aspectos psicossociais das mulheres

\footnotetext{
* Apoio: FAPESP, $\mathrm{CNPq}$

** E-mail: masantos@ffclrp.usp.br

n Submetido: 24/12/2015; Revisado: 19/12/2016; Aceito: 21/01/2017.
} 
acometidas, para subsidiar programas e intervenções que devem ser disponibilizados nos contextos dos serviços e no âmbito comunitário (Vidotti, Scorsolini-Comin, \& Santos, 2013). Para prover acompanhamento integral às pacientes, é necessário conhecer suas necessidades e de que modo os programas disponíveis contribuem para minimizar o impacto psicossocial da doença e os efeitos adversos de seus tratamentos (Caetano, Gradim, \& Santos, 2009).

Estratégias de atendimento psicológico podem ajudar pacientes a lidarem com o câncer e os efeitos dos tratamentos. Dentre as intervenções psicológicas mais utilizadas no cenário do câncer de mama, figuram o grupo de apoio, a psicoterapia de grupo suportivo-expressiva e o grupo psicoeducativo (Spiegel, 2001). Mas qual seriam os reais ganhos e benefícios proporcionados por essas intervenções em grupo? Em que circunstâncias e contextos específicos essas estratégias se aplicam? Essas indagações mostram a necessidade de delinear e analisar os desafios e possibilidades que se apresentam no conhecimento acumulado sobre as intervenções grupais utilizadas no cuidado psicossocial das mulheres mastectomizadas.

As mudanças decorrentes do tratamento realizado para o câncer de mama estão relacionadas, principalmente, à aparência física e a alterações psicossociais, o que inclui: manifestações de ansiedade, sintomas depressivos, diminuição da libido, problemas físicos, sociais e financeiros (Barrera \& Spiegel, 2014; Cesnik et al., 2013; Gonçalves, Tavares, Campana, Cabello, \& Shimo, 2012; Souza et al., 2014).

Durante toda a vida a mulher aprende que o corpo feminino é particularizado por alguns caracteres sexuais secundários que o diferenciam do masculino, sendo as mamas um dos mais proeminentes marcadores da diferença sexual. Assim, ao se submeter à mastectomia, a mulher vivencia a cirurgia como um ato agressivo, um ataque à estética e imagem corporal que construiu ao longo da vida, o que a faz perceber com estranheza o seu corpo após a intervenção cirúrgica (Ferreira \& Mamede, 2003; Peres \& Santos, 2012).

A convivência com outras mulheres que também retiraram a mama pode ser um elemento facilitador da aceitação da nova condição de mastectomizada (Caetano, Panobianco, \& Gradim, 2012). Assim, os programas de reabilitação que incluem grupos de apoio e de autoajuda são recursos importantes que podem auxiliar na compreensão dos problemas enfrentados e no alívio de parte substancial dos sintomas (Barbosa, Ximenes, \& Pinheiro, 2004; Fernandes, Rodrigues, \& Cavalcanti, 2004; Oliveira, Souza, \& Fernandes, 2008).

A utilização de grupos - de apoio, psicoeducativo ou de autoajuda - tem uma tradição já consolidada no contexto da saúde (Goldstein, \& Frantsve, 2009). Diversos estudos exploraram o potencial terapêutico das intervenções psicossociais em grupo (Boesen et al., 2011; Glaser \& Glassman, 2014; Hamama-Raz, Perry, Pat-Horenczyk, Bar-
Levav, \& Stemmer, 2012; Machavoine, 2010; Rowland et al., 2009; Spiegel, 2001; Vilhauer, McClintock, \& Matthews, 2010), com resultados majoritariamente positivos. O grupo auxilia suas integrantes a manter a esperança em face à doença grave, na medida em que oferece apoio e informação de forma ativa, contribuindo para reduzir o sentimento de isolamento e os níveis de estresse, além de ajudar as mulheres a desenvolver habilidades de resolução de problemas.

Para Cavalcanti, Fernandes e Rodrigues (2002), o grupo de apoio objetiva criar condições favoráveis de interação entre as mulheres com câncer de mama, estimulando reflexões sobre eventos de vida diários, especialmente as repercussões psicossociais da mastectomia, além de promover a melhora da autoestima e da qualidade de vida. O formato grupal favorece condições que proporcionam vivências que facilitam a troca de experiências e a catarse, fatores terapêuticos importantes do suporte oferecido para a reabilitação psicossocial.

O suporte do grupo torna-se, assim, importante para a reabilitação psicossocial de mulheres que tiveram ou ainda enfrentam o câncer de mama, contribuindo também para seu desenvolvimento e crescimento pessoal (Martins \& Peres, 2014). Ressalta-se que a assistência a essas mulheres não deve focalizar apenas a doença e a reabilitação física, deve abranger um contexto mais amplo da vida da mulher, que envolve também seus aspectos psicológicos, culturais, educacionais, econômicos e sociais (Caetano et al., 2012).

Estudo de metanálise sugere que os tratamentos de curta duração, com foco no enfrentamento - ou seja, que buscam aumentar o senso de controle que o indivíduo tem sobre si mesmo -, podem ser mais adequados para pacientes com câncer de mama descoberto precocemente, pois, nesse momento, a perturbação psicológica ou psiquiátrica desencadeada afeta a qualidade de vida dessas mulheres e pode comprometer a adesão ao tratamento. Lazarus e Folkman (1984) definem enfrentamento como as ações e estratégias cognitivas e comportamentais utilizadas pelo indivíduo frente a situações estressantes - como, por exemplo, uma doença grave como o câncer de mama. Essas situações desafiadoras podem ter origem tanto em demandas internas como externas, que ultrapassam o repertório do indivíduo, acarretando estados emocionais desconfortáveis e desequilíbrio pessoal, físico e social. Já as pacientes com câncer avançado parecem se beneficiar mais de intervenções de longo prazo, que enfatizam o apoio recebido por meio dos grupos (Naaman, Radwan, Fergusson, \& Johnson, 2009).

Dado que as pacientes com câncer de mama estão, atualmente, sobrevivendo por mais tempo com a doença ou livre de sintomas e, portanto, convivendo com a possibilidade de recidiva, abordar questões que estejam relacionadas à qualidade de vida assume um valor significativo. Essa abordagem também pode ser feita de forma eficiente por meio dos grupos de apoio (Naaman et al., 2009). 
As evidências existentes em relação aos potenciais benefícios oferecidos pelos grupos são fortes o suficiente para justificar a indicação do apoio do grupo psicossocial para mulheres com câncer de mama avançado, especialmente se o suporte é de longo prazo e se as pacientes mostram-se angustiadas no início da intervenção (Goodwin et al., 2001). No entanto, no cenário brasileiro, há poucos estudos que tenham se dedicado a investigar essa questão. Revisão da produção científica nacional identificou apenas 15 artigos - de um total de 101, localizados preliminarmente -, publicados entre 2001 e 2011. Segundo os autores, esses estudos indicam que a intervenção com grupos de apoio ainda é pouco difundida no contexto brasileiro (Leite \& Peres, 2013).

Essas considerações justificam o interesse em desenvolver uma revisão integrativa para avaliar a produção científica e que ela seja abrangente, incluindo tanto a literatura nacional como a internacional, sobre grupos de reabilitação voltados para mulheres com câncer de mama. A realização de um levantamento do conhecimento produzido na área pode subsidiar a prática clínica do profissional de saúde, bem como contribuir para levantar as necessidades de novas pesquisas e, assim, auxiliar no desenvolvimento de futuras investigações.

De modo geral, a literatura tem evidenciado que a participação nos grupos de apoio de fato auxilia na reabilitação psicossocial e no tratamento, contribuindo para potencializar o enfrentamento das situações de estresse e do medo da recidiva (Spiegel, 2001). Os estudos mostram que, depois de serem expostas às intervenções psicossociais, as mulheres apresentaram melhora na intensidade dos sintomas relacionados ao estresse, assim como no contato com a rede de amigos e familiares (Gomes, Panobianco, Ferreira, Kebbe, \& Meirelles, 2003; Yavuzsen, Karadibak, Cehreli, \& Dirioz, 2012). Os grupos também podem ajudar a amenizar as manifestações de ansiedade, depressão, raiva e hostilidade, que podem advir da descoberta do diagnóstico ou da cirurgia para retirada da massa tumoral (Gomes et al., 2003). Mas permanece em aberto - devido aos resultados dos estudos serem ainda inconclusivos - se a intervenção psicossocial em grupo pode ou não contribuir para aumentar a sobrevida em pacientes com câncer de mama (Goodwin, 2005).

Apesar dos avanços obtidos, ainda são escassos os estudos que buscam mapear e sistematizar o conhecimento atual sobre a temática dos grupos de apoio no câncer de mama. Definir o estado da arte nessa área de conhecimento é importante para identificar lacunas e definir prioridades de novas pesquisas. Frente ao exposto, este estudo teve por objetivo analisar a produção científica nacional e internacional sobre a temática dos grupos de reabilitação psicossocial para mulheres com câncer de mama, mais especificamente, os benefícios e limitações identificados, os temas norteadores da intervenção e a eficácia das intervenções realizadas.

\section{MÉTODO}

Esta é uma pesquisa documental, descritiva e retrospectiva, que teve como recorte temporal o período entre 2000 e 2015. A revisão integrativa foi escolhida como enfoque metodológico, pois propicia reunir vários estudos realizados anteriormente na temática de interesse, colocando em discussão seus métodos, fontes, objetivos e resultados, o que permite extrair conclusões a respeito do campo de conhecimento demarcado (Broome, 2000). Segundo Whittemore e Knafl (2005),

revisões integrativas são o método mais amplo de revisão de literatura, permitindo a inclusão simultânea de estudos experimentais e não-experimentais, a fim de compreender de forma mais completa o fenômeno investigado. Revisões integrativas podem também combinar os dados de pesquisas teóricas e empíricas, além de servir a uma vasta gama de propósitos, tais como: definir conceitos, revisar teorias e mostrar evidências. (p. 547)

Tendo em vista o objetivo de sumarizar o conhecimento produzido no decorrer de uma década e meia, optou-se por esse método de pesquisa porque ele possibilita a busca, avaliação crítica e síntese das evidências disponíveis sobre o tema investigado, sendo que o seu produto final é o estado atual do conhecimento do tema investigado, bem como a implementação de intervenções eficazes na assistência à saúde e a possível redução de custos, além da identificação de lacunas que podem nortear o desenvolvimento de pesquisas futuras (Cesnik \& Santos, 2012a; Mendes, Silveira, \& Galvão, 2008).

O estudo de revisão integrativa da literatura foi orientado pela seguinte questão norteadora: $\mathrm{O}$ que foi produzido na literatura a respeito dos grupos de apoio para mulheres com câncer de mama nos últimos anos?. A coleta dos dados foi realizada em periódicos indexados em bases regionais e internacionais de dados bibliográficos, a saber: LILACS, PubMed, PsycINFO e CINAHL. Essas bases indexadoras foram escolhidas por sua destacada importância em distintas áreas de produção do conhecimento científico, como medicina, psicologia e enfermagem.

Para alcançar o objetivo do estudo, foram seguidos os seguintes passos metodológicos, propostos por Broome (2000), para guiar a revisão da literatura: (a) realização de um levantamento sistematizado das publicações nacionais e internacionais sobre câncer de mama e grupos de apoio; (b) identificação dos autores, tipos de pesquisa, ano de publicação, periódicos nos quais foram veiculados tais 
artigos, país de origem das publicações, idioma em que foram redigidos, objetivos e resultados obtidos e (c) análise descritiva dos resultados e avaliação crítica das contribuições oferecidas para a produção de conhecimento sobre esse tema.

A coleta de dados foi realizada no período de março a julho de 2015. Foram pesquisados os artigos indexados com as seguintes palavras-chave: breast neoplasms; neoplasias da mama; self-help groups; grupos de auto-ajuda; psicoterapia de grupo e psycotherapy, group. Esses descritores foram escolhidos de acordo com sua disponibilidade no DECS (Descritores em Ciências da Saúde). A busca foi realizada por meio do sistema SIBI da Universidade de São Paulo, uma rede de serviços que possui um catálogo online que franqueia o acesso às bases de dados e conteúdos dos periódicos indexados, possibilitando a obtenção, na íntegra, dos artigos publicados em revistas disponibilizadas no sistema.

$\mathrm{Na}$ pesquisa bibliográfica, foram considerados como critérios de inclusão para busca dos artigos os seguintes parâmetros: (a) artigos circunscritos ao tema do câncer de mama feminino; (b) redigidos na língua inglesa, portuguesa ou espanhola; (c) publicados entre janeiro de 2000 e maio de 2015; (d) que apresentavam resultados empíricos e (e) que disponibilizavam o resumo nas bases de dados.
Como critérios de exclusão, estabeleceram-se os seguintes limites: (a) apresentação sob formato de dissertação, tese, capítulo de livro, livro, manual, editorial, resenha, carta, comentário, notícia ou crítica e (b) estudos de revisão da literatura.

Após a leitura dos resumos, procedeu-se a recuperação dos artigos selecionados, de acordo com os critérios de inclusão e exclusão estabelecidos. Esses artigos, recuperados na íntegra, constituíram o corpus da pesquisa. Após ler os estudos, procedeu-se a extração dos dados de interesse para a revisão. Utilizou-se, para tanto, um formulário apropriado para esse fim, sistematizado em estudos anteriores (Cesnik \& Santos, 2012b; Peres \& Santos, 2009; Santos \& PereiraMartins, 2016; Santos, Santos, \& Vieira, 2014).

Em seguida, as informações extraídas das publicações foram organizadas e submetidas à análise de conteúdo (Minayo, 2008), com o intuito de extrair os principais temas abordados pelas publicações. Essa análise ocorreu em três etapas: (a) pré-análise, (b) exploração do material e (c) tratamento dos resultados obtidos. Os dados foram organizados de acordo com os conteúdos prevalecentes no corpus investigado e analisados criteriosamente de acordo com os parâmetros preconizados pela literatura.

\section{RESULTADOS E DISCUSSÃO}

A amostra final foi composta por artigos indexados nas quatro bases de dados consultadas e que preencheram os critérios de inclusão utilizados para qualificar as publicações no processo de busca bibliográfica. Dos 1492 estudos listados, 40 satisfizeram esses critérios. Descartando-se os artigos não disponíveis na íntegra e os repetidos em mais de uma base de dados, 28 foram selecionados conforme os critérios preestabelecidos, constituindo o corpus deste estudo. A Figura 1 apresenta o flowchart com a descrição detalhada do processo de refinamento da busca e das

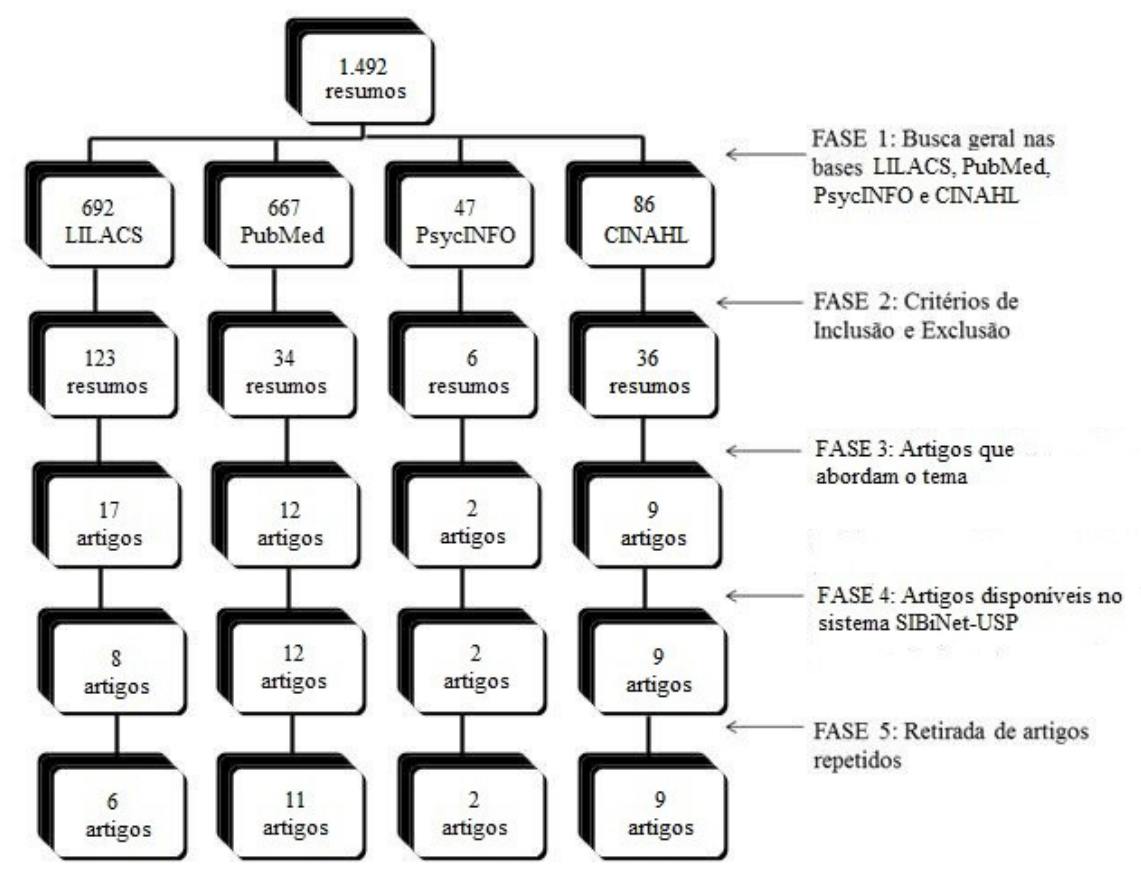

Figura 1. Fluxograma das fases do processo de seleção dos artigos da revisão integrativa, de acordo com o Preferred Reporting Items for Systematic Reviews and Meta-Analyses - PRISMA (Mother, Liberati, Tetzlaff, \& Altman, 2009). 
sucessivas etapas que permitiram a depuração da amostra de artigos.

Do total de artigos encontrados nos diferentes arranjos de descritores, houve diferença na efetividade das combinações de palavras-chave de acordo com a base de dados consultada. Assim, a combinação breast neoplasms e self-help groups, bem como a combinação breast neoplasms e psycotherapy, group mostraram-se as mais promissoras para as bases PubMed, CINAHL e LILACS, enquanto que as combinações neoplasias de mama egrupos de auto-ajuda e neoplasias de mama e psicoterapia de grupo se mostraram mais eficazes na busca empreendida na base de dados LILACS. Já as combinações breast neoplasms e psychotherapy group e breast neoplasms e self-help groups foram as únicas combinações que surtiram resultado na base PsycINFO, sendo que apenas a segunda combinação permitiu recuperar artigos que foram de fato selecionados. Não foi utilizado nas buscas o termo grupo de apoio, por não estar indexado como DECs. Esses resultados podem ser melhor apreciados na Tabela 1.

Os artigos selecionados foram publicados em 22 revistas científicas distintas. O periódico Psycho-Oncology destacouse como o que concentra o maior volume de publicações referentes à temática dos grupos de apoio no câncer de mama, com três artigos (13.6\%) dedicados a esse objeto de estudo no período estabelecido para análise. Essa revista é dedicada a explorar a interface entre Psicologia e câncer, o que acaba contribuindo para a publicação de pesquisas relacionadas à temática focalizada nesta revisão.

Além desse periódico, outros quatro concentraram dois artigos cada $(9 \%)$ do total do corpus de análise: Patient Education and Counseling, American Cancer Society, Journal of Clinical Oncology e Journal of Psychosocial Oncology. Outras 17 revistas científicas estão representadas no corpus, veiculando apenas um artigo cada, sendo quatro nacionais. A maioria desses periódicos tem caráter multidisciplinar e divulgam conhecimento científico arbitrado, o que contribui para a construção da complexidade do saber na área do câncer de mama e dos grupos de apoio.
Quando investigadas as áreas focalizadas pelos periódicos que veiculam os artigos selecionados, observouse que muitas revistas se concentram em temáticas multidisciplinares ou interdisciplinares, que atuam em temas focados no câncer ou, de modo mais amplo, na saúde. O caráter multidisciplinar pode ser visto também nos autores dos artigos, já que os estudos foram assinados por profissionais de diferentes áreas da saúde. Dos primeiros autores, 10 são médicos, três psicólogos, dois professores (sem especificar sua formação profissional), um enfermeiro e um jornalista. Em 11 casos não foi possível identificar as profissões dos primeiros autores. Em relação aos últimos autores de cada artigo, tem-se que 13 são médicos, dois enfermeiros e dois psicólogos. Também não foi possível identificar as profissões dos últimos autores de 11 artigos.

Quanto ao país de origem do primeiro autor de cada artigo, 28,6\% dos autores eram oriundos dos Estados Unidos, $17,8 \%$ do Canadá, $14,3 \%$ do Brasil, 7,1\% dos Países Baixos e o restante da amostra foi composta por um estudo de cada país: Reino Unido, Nova Zelândia, Israel, Noruega, França, Suécia, Turquia, Alemanha e China. Já quanto ao país de origem do último autor, 32,1\% são dos Estados Unidos, $17,8 \%$ do Canadá, $14,3 \%$ do Brasil, 7,1\% dos Países Baixos e o restante da amostra foi composta por um estudo de cada país: Reino Unido, Nova Zelândia, Israel, Noruega, França, Suécia, Turquia e Alemanha.

Em relação aos autores brasileiros (oito), três eram filiados à Universidade Federal do Ceará (UFC), dois à Universidade de São Paulo (USP), dois à Universidade Sagrado Coração (USC), do município de Bauru (SP), e um à Faculdade Integrada do Ceará (FIC). Desses pesquisadores, um fazia parte do departamento de psicologia, um do departamento de enfermagem materno-infantil e saúde pública, um do departamento de enfermagem e em seis casos não foi possível identificar o departamento de origem. Além disso, foi possível constatar que quatro autores realizaram a pesquisa de campo na cidade de Fortaleza (CE), dois autores em Ribeirão Preto (SP) e dois em Bauru (SP).

Delinear os temas subjacentes aos artigos revisados permite definir os eixos temáticos que nortearam o

Tabela 1

Distribuição Numérica de Publicações Encontradas (E) e Selecionadas (S) nas Bases Indexadoras Consultadas

\begin{tabular}{|c|c|c|c|c|c|c|c|c|c|c|}
\hline \multirow[t]{2}{*}{ Palavras-chave } & & & \multicolumn{2}{|c|}{ PubMed } & \multicolumn{2}{|c|}{ LILACS } & \multicolumn{2}{|c|}{ PsycINFO } & \multicolumn{2}{|c|}{ CINAHL } \\
\hline & & & $\mathrm{E}$ & $\mathrm{S}$ & $\mathrm{E}$ & S & $\mathrm{E}$ & S & $\mathrm{E}$ & $\mathrm{S}$ \\
\hline "Neoplasias da mama" & $\mathrm{e}$ & "Grupos de autoajuda" & 0 & 0 & 7 & 3 & 0 & 0 & 0 & 0 \\
\hline "Breast neoplasms" & e & “Self-help groups" & 409 & 6 & 417 & 2 & 38 & 2 & 19 & 5 \\
\hline "Neoplasias da mama" & $\mathrm{e}$ & "Psicoterapia de grupo" & 0 & 0 & 268 & 1 & 0 & 0 & 0 & 0 \\
\hline \multirow[t]{2}{*}{ "Breast neoplasms" } & e & "Psycotherapy, Group" & 258 & 5 & 0 & 0 & 9 & 0 & 67 & 4 \\
\hline & & Total & 667 & 11 & 692 & 6 & 47 & 2 & 86 & 9 \\
\hline
\end{tabular}


desenvolvimento dos estudos, as preocupações que os motivaram e os potenciais benefícios e limitações dos grupos identificados pelas pesquisas, como pode ser visualizado na Tabela 2. Emergiram 11 categorias, que não são mutuamente excludentes, de maneira que um mesmo artigo poderia estar classificado em mais de um tema encontrado.

Os resultados mostram que um dos focos principais dos estudos recai sobre o ajustamento emocional, significando os benefícios de ordem emocional que as mulheres auferem no grupo de apoio, englobando 20 dos artigos selecionados. Nos estudos que abordam a questão do ajustamento, foi comum encontrar a constatação de que o fato de as mulheres poderem partilhar seus sofrimentos e informações sobre a doença durante a reunião de um grupo de pares ajuda a diminuir a tensão e a expectativa ansiosa frente ao desconhecido. Compartilhar suas vivências traz conforto e as deixa menos assustadas com relação a tudo o que está acontecendo em suas vidas desde que tomaram conhecimento do diagnóstico e que passaram a se submeter aos vários tratamentos.

Além disso, alguns estudos - encontrados na revisão e que serão detalhados a seguir - evidenciam que o apoio emocional e social pode contribuir para aumentar a sobrevida das mulheres com câncer de mama (Cameron et al., 2005; Geiger, Mullen, Sloman, Edgerton, \& Petitti, 2000; Pinheiro, Silva, Mamede, \& Fernandes, 2008). Também foi constatado que a depressão pode ser um desfecho resultante das preocupações relacionadas ao câncer, seus sintomas e tratamentos. Desse modo, conversar sobre esses tópicos pode abrandar a carga emocional decorrente dessas preocupações e, consequentemente, diminuir a vulnerabilidade à depressão nas mulheres com câncer de mama (Palesh et al., 2007). Os estudos são convergentes ao apontarem que os níveis de ansiedade declinam e são melhor controlados quando as mulheres frequentam grupos de apoio (Classen et al., 2008; Dolbeault et al., 2009; Spiegel et al., 2007).

O segundo foco principal foi o bem-estar e ajustamento psicossocial, englobando o bem-estar físico e social das mulheres com câncer de mama, presente em 20 dos artigos revisados. Foi relatado que mulheres que participam de grupos de apoio apresentam melhora na qualidade de vida e, de modo mais amplo, também aferem ganhos em termos de "qualidade psicológica", entendida pelos autores dos estudos como progressos na saúde mental, bem-estar psicológico e outros benefícios correlatos (Emslie et al., 2007; Kissane et al., 2007; Namkoong et al., 2010).

Outro estudo mostrou que as intervenções grupais que visam a promover melhora no bem-estar psicossocial e na adaptação de pacientes com câncer estão ganhando reconhecimento como ferramentas essenciais no campo da Psico-Oncologia (Cohen \& Fried, 2007). Mulheres que participam dos grupos e que têm mais dificuldades de ajustamento obtiveram benefícios psicossociais, enquanto que aquelas que não estavam tão angustiadas no início da intervenção não lograram os mesmos resultados (Goodwin
Tabela 2

Distribuição Percentual dos 11 Temas Identificados como Resultado da Intervenção em Grupo nos Artigos Revisados

\begin{tabular}{lcc}
\hline \multicolumn{1}{c}{ Temas identificados } & N & \% artigos \\
\hline Ajustamento emocional & 20 & 71,4 \\
Bem-estar e ajustamento & 20 & 71,4 \\
psicossocial & 13 & 46,4 \\
Estresse & 9 & 32,1 \\
Controle da dor & 7 & 25,0 \\
Limitações dos grupos & 7 & 25,0 \\
Sobrevivência & 7 & 25,0 \\
Materiais educativos / & 4 & 14,3 \\
psicoeducacionais & 2 & 7,1 \\
Empoderamento & 1 & 3,6 \\
Autoeficácia & 1 & 3,6 \\
Funcionamento psicossexual & Crescimento pós-traumático &
\end{tabular}

et al., 2001). Vos, Virssen, Garssen, Duivenvoorden e Haes $(2006,2007)$ relatam melhora no ajustamento psicossocial das mulheres, não importando se frequentavam o grupo há muito ou pouco tempo. Bordeleau et al. (2003) constataram que o ajustamento psicossocial contribui para melhorar a qualidade de vida das mulheres que frequentam grupos de apoio. Um dado a se reter é que, provavelmente, o grupo irá beneficiar mais as mulheres que manifestam níveis mais elevados de angústia (Goodwin et al., 2001), o que sugere que são pacientes que claramente têm indicação para receberem essa modalidade de intervenção.

O terceiro tema mais destacado foi o estresse, que englobou 13 dos artigos selecionados. Constatou-se que os grupos de apoio ajudam a reduzir o estresse emocional e a melhorar as habilidades das mulheres de lidar com os estressores e com a própria doença (Dolbeault et al., 2009; Stang \& Mittelmark, 2008; Vos et al., 2007). Por outro lado, também foi encontrado que mulheres com níveis de estresse muito elevados não auferem benefício algum desses grupos (Classen et al., 2008). O resultado desse último estudo é relevante, pois evidencia que há limitações na aplicação dos grupos de apoio que não dizem respeito à metodologia empregada para conduzir a intervenção ou programa de reabilitação psicossocial, mas sim a variáveis pessoais e características de personalidade das possíveis beneficiárias. Outro aspecto a ser assinalado é que os resultados de Classen et al. estão em contradição com os achados de Goodwin et al. (2001), que, como referido anteriormente, detectaram que as participantes mais angustiadas se beneficiam mais dos grupos.

O quarto tema foi controle da dor, englobando nove estudos. A dor física é definida como percepção sensorial relacionada a fenômenos emocionais e a componentes da personalidade das mulheres, por isso, é importante abordar esse sintoma nos grupos de apoio (Dolbeault et al., 2009; Pinheiro et al., 2008). Goodwin et al. (2001) relatam que 
a melhora nos sintomas de dor só ocorre nas mulheres que estão mais angustiadas. Já o estudo de Spiegel et al. (2007) comprovou que os níveis de dor decrescem quando as mulheres participam dos grupos, em qualquer condição. Essa contradição entre os resultados obtidos por Goodwin et al. (2001) e Spiegel et al. (2007) merece ser melhor explorada por estudos futuros.

O quinto tema concerne às limitações dos grupos. Esse tema englobou sete dos artigos. No que tange ao enfrentamento, há evidência limitada de que o uso de serviços de apoio às pacientes com câncer de mama se relaciona à obtenção de maior suporte social e menor uso de estratégias de enfrentamento do tipo evitativo (Cameron et al., 2005). O referido estudo investigou a participação em um programa de apoio psicossocial e treinamento de habilidades de enfrentamento para mulheres recentemente diagnosticadas. Programas desse tipo e materiais informativos que promovam sua utilização podem atrair mais participantes se forem adaptados para concentrarse em resolver angústias relacionadas ao câncer, em vez de focarem na ansiedade geral ou na depressão.

O êxito desses programas parece depender também das habilidades do coordenador de priorizar participantes com tendência acentuada de evitar o contato com os problemas, abordar o papel da função imunológica na progressão do câncer e satisfazer as necessidades específicas das participantes mais velhas. Como esses programas são menos atrativos para as pacientes que frequentam há mais tempo os grupos, é importante que se desenvolvam materiais informativos que enfatizem que a intervenção também é apropriada para essas mulheres. Cohen e Fried (2007) e Gray, Fitch, Davis e Phillips (2000) mostraram que as limitações dos grupos de apoio estão relacionadas ao pouco conhecimento e reduzida habilidade em coordenação de grupos de quem implementa os programas. Assim, as limitações não seriam intrínsecas à abordagem em grupo, mas à falta de preparo no manejo de grupos e/ou baixa competência dos profissionais que aplicam intervenções grupais.

O sexto tema encontrado foi sobrevivência, que também abarcou sete dos artigos. Estudo mostrou que a psicoterapia de grupo não contribuiu para prolongar a sobrevida de mulheres com câncer de mama, porém ajudou a melhorar o humor e a manejar a dor (Goodwin et al., 2001). Em outro estudo, a análise multivariada confirmou que as pacientes escolhidas para receberem a intervenção tiveram um risco significativamente menor de recorrência da doença e também de morte em decorrência do câncer ou de qualquer outra doença (Andersen et al., 2008). Spiegel et al. (2007) não conseguiram replicar, em sua pesquisa posterior, a conclusão de seu estudo anterior, que mostrou aumento da taxa de sobrevivência entre as mulheres com câncer de mama que frequentaram o grupo de apoio durante um ano.

$\mathrm{O}$ sétimo tema diz respeito à oferta de materiais educacionais/psicoeducacionais, englobando temas relacionados ao aprendizado de atividades e informações sobre a doença e o tratamento, que abrangeu sete dos artigos selecionados. Os resultados do estudo de Santos, Prado, Panobianco e Almeida (2011) mostraram que o grupo promove um ambiente permissivo, que estimula a oferta de suporte social, compartilhamento de sentimentos, desenvolvimento de habilidades para o enfrentamento de situações difíceis ou adversas, educação em saúde, acesso à informação e discussão de questões existenciais. Oliveira et al. (2008) constataram que determinadas atividades educativas, como oficinas, realizadas nesses grupos trazem benefícios para as mulheres que os frequentam, em termos de equilíbrio psicossocial e qualidade de vida. Um dos estudos analisados confirmou que grupos que têm uma estruturação psicoeducativa são mais eficientes do que os grupos que não apresentam essa configuração (Classen et al., 2008).

O oitavo tema foi empoderamento, que, no contexto dos estudos revisados, tem por objetivo aumentar o senso de controle pessoal das pacientes, bem como promover um enfrentamento positivo e bem-estar psicológico. Esse tema abrangeu quatro das publicações selecionadas. $\mathrm{O}$ empoderamento é um conceito multidimensional, que envolve aspectos cognitivos, afetivos e de conduta. Segundo Kleba e Wendausen (2009), pode ser definido como o "aumento do poder, da autonomia pessoal e coletiva de indivíduos e grupos sociais nas relações interpessoais e institucionais, principalmente daqueles submetidos a relações de opressão, discriminação e dominação social” (p. 735).

Estudos realizados mostraram que o compartilhamento de sentimentos e dúvidas sobre a doença e suas terapêuticas reforçam a possibilidade de as mulheres recuperarem o controle sobre suas próprias vidas, em vez de se manterem em uma posição submissa frente aos profissionais da saúde. Essa postura ativa favorece o empoderamento e incentiva a adoção de atitudes positivas frente às adversidades, o que é desejável para que se possam fortalecer os comportamentos de busca de saúde (Santos et al., 2011; Stang \& Mittelmark, 2008). Nessa direção, Kissane et al. (2007) utilizaram um método de empoderamento para otimizar os benefícios dos grupos de apoio voltados às mulheres com câncer de mama.

O nono tema foi autoeficácia, definida como a crença do indivíduo na sua capacidade de produzir resultados desejáveis (Bandura, Pastorelli, Barbaranelli, \& Caprara, 1999), presente em dois estudos. Os efeitos da expressão e recepção de informações a respeito do tratamento sobre o bem-estar emocional mostram relação com a autoeficácia na saúde. Se a autoeficácia era alta, os efeitos eram positivos, e se era baixa, os efeitos eram negativos (Namkoong et al., 2010). Cohen e Fried (2007) corroboraram resultados de estudos anteriores, que demonstraram que a elevação na autoeficácia era acompanhada de melhora no estado de humor, ajustamento psicossocial e qualidade de vida em pacientes que participaram de grupos de apoio.

O décimo tema foi funcionamento psicossexual, que englobou um artigo. Vos et al. (2007) não encontraram mudanças significativas no funcionamento psicossexual 
das mulheres com câncer de mama que participavam de grupos de apoio.

O último tema encontrado foi crescimento póstraumático, conceito que engloba mudanças positivas na autopercepção, nas relações interpessoais e/ou na filosofia de vida após ter vivenciado um evento traumático (Tedeschi $\&$ Calhoun, 1996). Essa categoria compreendeu apenas um estudo. Kent et al. (2013) encontraram que o fato de participar de um programa de apoio e confiar nos profissionais de saúde foi associado ao maior crescimento pós-traumático. Como esse construto é definido como a mudança positiva que uma pessoa pode experimentar como resultado de seu processo de luta para sobreviver emocionalmente, a uma experiência traumática, pode-se afirmar que os grupos potencializam a elaboração psicológica do impacto dessas vivências e favorecem a assimilação das aprendizagens e benefícios que podem advir do contato com situações adversas. $\mathrm{Na}$ Figura 2, é possível observar a distribuição do total de artigos que compõem o corpus do estudo, de acordo com o ano de publicação.

Quanto ao idioma no qual os artigos foram publicados, verificou-se que $85.7 \%$ foram redigidos na língua inglesa, $10.7 \%$ na língua portuguesa e $3.6 \%$ na língua espanhola. Outros dados mais específicos sobre os artigos selecionados, como autores/ano de publicação, população estudada e delineamento de pesquisa podem ser encontrados na Tabela 3.

Alguns autores mostraram que a participação de mulheres com câncer de mama em grupos de apoio colabora significativamente para sua reabilitação integral, ou seja, aquela que contempla integralmente as necessidades físicas, psíquicas e sociais das pacientes, o que mostra que esses grupos não se limitam somente a fornecer ajuda psicológica.
A convivência com outras mulheres que também precisaram retirar a mama é um elemento que auxilia na aceitação de sua condição de ser mastectomizada e no entendimento dos problemas resultantes da intervenção cirúrgica, pois o período que sucede ao diagnóstico de câncer de mama é especialmente difícil e emocionalmente mobilizador. A mulher acometida necessita receber suporte social e familiar para enfrentar as condições adversas e se envolver nos desafios da readaptação, aprendendo a lidar de forma efetiva com os problemas que afetam seu cotidiano em decorrência da nova condição de mastectomizada (Caetano et al., 2012; Silva \& Santos, 2010).

Estudo qualitativo objetivou compreender o sentimento da mulher mastectomizada desde o diagnóstico até a possibilidade da recorrência do câncer de mama (Pinho, Campos, Fernandes, \& Lobo, 2007). Participaram 15 mulheres integrantes de um grupo de autoajuda. Os resultados mostraram que o significado do grupo de autoajuda em suas vidas era mediado pelos sentimentos desencadeados com a descoberta do câncer e a realização da mastectomia. $\mathrm{O}$ impacto das adversidades é atenuado pela expectativa de cura que, no entanto, convive com o temor da recorrência. As autoras constataram que o diagnóstico do câncer de mama tende a ser vivido por essas mulheres como uma experiência irreal - ou até mesmo surreal - em um primeiro momento. A doença é percebida como letal e assustadora, desencadeando sentimentos disfóricos como surpresa, medo, desamparo e desespero. Nesse cenário, o grupo de autoajuda surge como um potente recurso de apoio, por meio do qual as mulheres podem interagir, trocar e compartilhar experiências, tornando-se mais conscientes e

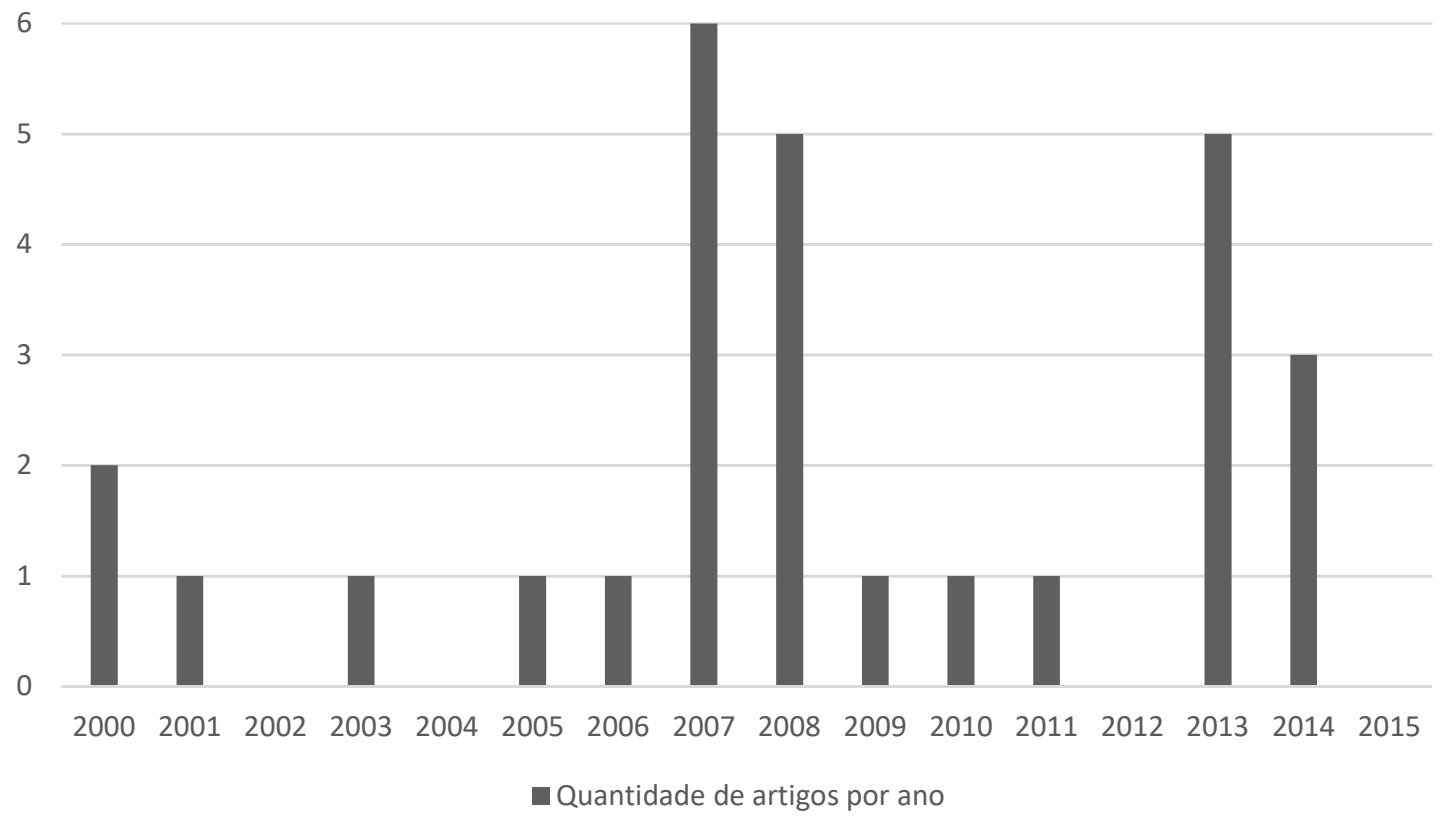

Figura 2. Distribuição dos artigos selecionados de acordo com o ano de publicação 
Tabela 3

Caracterização dos Artigos Recuperados segundo Autores/Ano, População Estudada e Delineamento do Estudo $(n=28)$

\begin{tabular}{ll} 
Autores & População estudada \\
\hline Mulheres com câncer de mama recém-diagnosticado, inscritas nos sites de um programa de
\end{tabular}

Geiger et al. (2000) apoio e informação $(\mathrm{n}=111)$ e uma amostra aleatória de mulheres com câncer de mama não cadastradas nesses sites $(\mathrm{n}=277)$.

Gray et al. (2000)

Quatro grupos de apoio para mulheres com câncer de mama, recrutadas de comunidades de Ontário, Canadá.

235 mulheres com câncer de mama, as quais se esperava que sobreviveriam pelo menos

Goodwin et al. (2001) por três meses, distribuídas em uma proporção de 2:1 para um grupo de intervenção de apoio social semanal (158 mulheres) e para um grupo controle que não recebeu a intervenção (77 mulheres).

Bordeleau et al. (2003)

235 mulheres de sete centros canadenses de tratamento oncológico foram distribuídas aleatoriamente em grupos de intervenção psicossocial padronizada (terapia de apoio suportivo-expressiva), com frequência semanal, e grupos controle.

Cameron et al. (2005) Das 149 mulheres convidadas a participar, 110 mulheres (74\%) concordaram, 26 mulheres (17\%) declinaram e 13 (9\%) não responderam.

67 mulheres em estágio inicial de câncer de mama foram aleatoriamente designadas para participarem de um grupo de intervenção, que começou quatro meses depois da cirurgia, ou para o mesmo grupo de intervenção, mas que teve início pelo menos três meses depois do primeiro.

Vos et al. (2006)

114 pacientes com câncer de mama em estágio inicial foram aleatoriamente designadas

Cohen \& Fried (2007) para grupo de terapia cognitivo-comportamental (grupo de intervenção) versus grupo de relaxamento e imaginação guiada (grupo controle).

De um total de 203 mulheres oriundas do oeste da Escócia, com média de idade de

Emslie et al. (2007) 53 anos, que haviam se submetido a tratamento para câncer de mama em estágio inicial, 36 participaram de sete grupos focais, com cinco mulheres em média em cada grupo.

De 485 mulheres com câncer de mama avançado, recrutadas entre 1996 e 2002, 227 (47\%) concordaram em participar e foram randomizadas, em um intervalo médio de 10 meses desde a recorrência do câncer, em uma proporção de 2:1, para um programa de intervenção de um ano ou mais de terapia de grupo suportivo-expressiva semanal, além de três sessões de terapia de relaxamento (147 mulheres), enquanto que as participantes do grupo controle receberam apenas as três sessões de terapia de relaxamento ( 80 mulheres).

Palesh et al. (2007)

93 mulheres com câncer de mama, que completaram questionários que avaliavam sintomas de depressão, dor, estresse e distúrbios do sono, durante quatro, oito e 12 meses.

125 mulheres com câncer de mama primário $(\mathrm{n}=122)$ ou localmente recorrente $(\mathrm{n}=3)$

Spiegel et al. (2007) foram selecionadas, aleatoriamente, para o grupo de apoio $(\mathrm{n}=64)$ ou para a condição controle $(n=61)$.

67 mulheres com bom ajustamento psicossocial, que haviam sido submetidas à cirurgia havia

Vos et al. (2007) pelo menos quatro meses antes do início do estudo, foram randomizadas em uma das duas condições: participação na psicoterapia de grupo ou no grupo de apoio social.

Andersen et al. (2008) 227 pacientes que foram tratadas cirurgicamente para câncer de mama.

Classen et al. (2008)

353 mulheres que estavam no período de um ano do diagnóstico de câncer de mama primário foram distribuídas aleatoriamente para participarem de um programa de 12 semanas de terapia de grupo suportivo-expressiva ou um grupo controle educacional.

População indefinida de mulheres mastectomizadas cadastradas em um grupo de apoio/ suporte. Relato de experiência, com objetivo de descrever a dinâmica do serviço,

Oliveira et al. (2008) desenvolvido a partir de consultas recorrentes aos documentos, livro de atas, fotografias, gravações e trabalhos relacionados com o grupo desde 1998.

Pinheiro et al. (2008) 30 mulheres mastectomizadas, assistidas em seis grupos de apoio existentes no município de Fortaleza, Ceará.

18 mulheres, distribuídas em três grupos de cinco a sete integrantes, participaram de Stang \& Mittelmark discussões em grupo de autoajuda, conduzido por profissionais com frequência semanal, (2008) por aproximadamente quatro meses. Durante o processo, cada grupo foi entrevistado por três vezes em grupos focais.

203 mulheres arregimentadas após tratamento primário do câncer de mama foram designadas

Dolbeault et al. (2009) aleatoriamente para um grupo de tratamento (intervenção psicoeducativa) ou para um grupo controle, constituído por pacientes da lista de espera.

Namkoong et al. (2010)
177 mulheres com câncer de mama, selecionadas por meio de um programa eletrônico de saúde (eHealth) com grupo de discussão.
Delineamento do estudo

Qualitativo, nãoexperimental e descritivo

Qualitativo, nãoexperimental e descritivo

Método misto, nãoexperimental e descritivo

Qualitativo, nãoexperimental e descritivo

Quantitativo, nãoexperimental e descritivo

Quantitativo, nãoexperimental e descritivo

Qualitativo, nãoexperimental e descritivo

Qualitativo, nãoexperimental e descritivo

Método misto, nãoexperimental e descritivo

Método misto, nãoexperimental e descritivo

Qualitativo, nãoexperimental e descritivo

Quantitativo, nãoexperimental e descritivo

Quantitativo, nãoexperimental e descritivo

Método misto, nãoexperimental e descritivo

Documental, qualitativo, não-experimental e descritivo

Qualitativo, nãoexperimental e descritivo

Qualitativo, nãoexperimental e descritivo

Quantitativo, nãoexperimental e descritivo

Quantitativo e qualitativo, não-experimental e descritivo 
Tabela 3

Cont.

\begin{tabular}{|c|c|c|}
\hline Autores & População estudada & Delineamento do estudo \\
\hline Santos et al. (2011) & $\begin{array}{l}\text { Mulheres com câncer de mama que participam de um grupo de apoio oferecido por um } \\
\text { serviço de reabilitação psicossocial. Esse serviço acolhe, em média, } 100 \text { casos novos por } \\
\text { ano e presta assistência a cerca de } 200 \text { mulheres registradas em anos anteriores. }\end{array}$ & $\begin{array}{l}\text { Qualitativo, não- } \\
\text { experimental e descritivo }\end{array}$ \\
\hline $\begin{array}{l}\text { Björneklett et al. } \\
(2013)\end{array}$ & $\begin{array}{l}\text { De um total de } 382 \text { pacientes recém-diagnosticadas com câncer de mama, } 191 \text { foram } \\
\text { randomizadas para um grupo de intervenção ou um grupo controle de rotina. O primeiro } \\
\text { recebeu intervenção de apoio na residência por uma semana, seguida de quatro dias de } \\
\text { seguimento dois meses mais tarde. A intervenção incluiu sessões informativas / educacionais, } \\
\text { práticas de relaxamento, visualização mental e comunicação não-verbal. }\end{array}$ & $\begin{array}{l}\text { Quantitativo, não- } \\
\text { experimental e descritivo }\end{array}$ \\
\hline Carlson et al. (2013) & 271 mulheres diagnosticadas com câncer de mama com estadiamento de I a III. & $\begin{array}{l}\text { Quantitativo, não- } \\
\text { experimental e descritivo }\end{array}$ \\
\hline Cheng et al. (2013) & $\begin{array}{l}29 \text { mulheres chinesas com câncer de mama, selecionadas dentre as que participavam } \\
\text { de uma organização local de autoajuda para o câncer. }\end{array}$ & $\begin{array}{l}\text { Qualitativo, não- } \\
\text { experimental e descritivo }\end{array}$ \\
\hline Kent et al. (2013) & 604 mulheres com câncer de mama, com idades entre 40 e 64 anos. & $\begin{array}{l}\text { Quantitativo, não- } \\
\text { experimental e descritivo }\end{array}$ \\
\hline Simeão et al. (2013) & 50 mulheres diagnosticadas com câncer de mama com idade média de 57,2 anos. & $\begin{array}{l}\text { Quantitativo transversal e } \\
\text { descritivo }\end{array}$ \\
\hline Rizalar et al. (2014) & $\begin{array}{l}100 \text { mulheres voluntárias, diagnosticadas com câncer de mama nos últimos seis meses, } \\
\text { vinculadas a uma unidade de quimioterapia ambulatorial de um hospital do norte da Turquia. }\end{array}$ & $\begin{array}{l}\text { Quantitativo, não- } \\
\text { experimental e descritivo }\end{array}$ \\
\hline Beutel et al. (2014) & $\begin{array}{l}157 \text { pacientes com câncer de mama em comorbidade com depressão, distribuídas } \\
\text { randomicamente para grupo de psicoterapia psicodinâmica breve (grupo de intervenção, } \\
\mathrm{N}=78 \text { ) ou para grupo de tratamento regular (grupo controle, } \mathrm{N}=79 \text { ). }\end{array}$ & $\begin{array}{l}\text { Quantitativo, não- } \\
\text { experimental e descritivo }\end{array}$ \\
\hline $\begin{array}{l}\text { Shannonhouse et al. } \\
\text { (2014) }\end{array}$ & $\begin{array}{l}23 \text { mulheres com câncer de mama participaram do grupo de apoio FYNN } \\
\text { (Finding Your New Normal), das quais } 14(61 \%) \text { concordaram em participar do estudo. }\end{array}$ & $\begin{array}{l}\text { Método misto, não- } \\
\text { experimental e descritivo }\end{array}$ \\
\hline
\end{tabular}

somando forças para superar juntas o temor da recorrência do câncer de mama.

Em estudo de abordagem qualitativa, Cavalcanti et al. (2002) entrevistaram 10 integrantes de um grupo de apoio oferecido no contexto da reabilitação da mulher mastectomizada. Os significados atribuídos ao grupo foram: partilha de sentimentos e necessidades, mecanismos de solução de problemas, ambiente educativo, espaço interativo que oportuniza amizades e troca de experiências. Pôde-se perceber que a comunicação no âmbito do grupo é um mecanismo muito utilizado pelas mastectomizadas para reforçar e elevar sua autoestima, melhorar a qualidade de vida e obter maior equilíbrio interior.

Estudo que buscou compreender o significado dos grupos de apoio na vida das mulheres com câncer de mama constatou que a participação no grupo proporciona cuidado diferenciado, promovendo a socialização das experiências vividas com a doença e seus tratamentos (Pinheiro et al., 2008), bem como o combate ao medo da recidiva, que permanece como um fantasma que paira sobre a vida das sobreviventes. Outro estudo, que também identificou os significados do grupo de autoajuda na reabilitação de mulheres mastectomizadas, mostrou que as participantes atribuem grande importância às atividades grupais e que os grupos funcionam como centros de apoio psicológico, terapêutico, educativo e interativo (Fernandes, Cavalcanti, Bonfim, \& Melo, 2005).

Conviver com uma doença estigmatizante como o câncer de mama obriga a mulher a enfrentar preconceitos e a lidar com sentimentos negativos, que refletem alto grau de incerteza e incontrolabilidade em relação ao próprio viver (Peres \& Santos, 2007, 2009; Rossi \& Santos, 2003; Silva \& Santos, 2010). Por outro lado, ver-se face a face com a adversidade deflagra um processo de interpretação da experiência de viver com a doença e seus tratamentos. Ser revestida com um significado favorece com que a experiência seja subjetivada e integrada. Com o auxílio do grupo de pares, podem ser ativados processos de significação de conceitos, crenças, sentimentos, atitudes e valores de vida, que possibilitam elaborar uma nova percepção sobre a doença, até então vista como degradante e incurável (Pinho et al., 2007; Silva \& Santos, 2008). Os benefícios da atividade de grupo também foram relacionados à percepção de mudanças na aparência física e nos cuidados pessoais, bem como na qualidade de vida (Fernandes, Barbosa, \& Silva, 2002)

Goodwin et al. (2001) tomaram como ponto de partida resultados de pesquisa que sugeriam que a participação nos grupos não só surtia benefícios psicológicos, como também resultava no prolongamento do tempo de sobrevivência. Examinando mulheres com câncer de mama metastático, esses autores encontraram que, em comparação com o grupo controle, as mulheres que receberam a intervenção (psicoterapia suportivo-expressiva) mostraram marcada melhora nos sintomas psicológicos (estado de humor) e na percepção de dor (relataram sentir menos dor). As participantes que tinham maiores níveis de estresse foram beneficiadas, ao contrário das que estavam menos estressadas. Contudo, a intervenção psicológica não 
prolongou a sobrevivência e a média de sobrevida foi de 17 meses para ambos os grupos.

Além disso, embora se observe que muitas mulheres buscam os grupos de apoio, outras, que se sentiam desconfortáveis em participar dessa experiência ou que não tiveram acesso a tais grupos, expressaram culpa, pois acreditavam que poderiam não estar fazendo todo o possível para prolongar sua sobrevivência. Esse sentimento de culpa muitas vezes foi intensificado por membros da família e amigos que, embora bem-intencionados, com frequência exerceram pressão sobre as mulheres para que elas se empenhassem em buscar ajuda nos grupos de apoio (Goodwin, 2005).

Apesar de serem encontrados relatos da potencial contribuição para o prolongamento do tempo de sobrevida em mulheres com câncer de mama avançado, diversos estudos mostraram que as intervenções psicossociais não contribuíram para aumentar a curva de sobrevivência. Como já mencionado, Goodwin et al. (2001) - e também Kissane et al. (2007) e Spiegel et al. (2007) - não confirmaram a melhora na taxa de sobrevivência em decorrência da participação em grupo de apoio social ou terapia de grupo suportivo-expressiva. Esses estudos contrastam com os achados obtidos por outro estudo que, em relação aos potenciais benefícios da intervenção psicossocial em grupo, comprovou que, após 11 anos, em média, de acompanhamento, a recorrência do câncer de mama acometeu 62 das $212(29 \%)$ pacientes e a morte ocorreu em 54 (24\%) das participantes (Andersen et al., 2008).

Como previsto pelos autores, as pacientes do grupo de intervenção tiveram uma redução significativa do risco de recorrência e de morte (por todas as causas) em comparação com aquelas que não participaram do grupo. No referido estudo, a intervenção psicológica foi conduzida em pequenos grupos e incluiu estratégias de redução de estresse, melhora do estado de humor, promoção de comportamentos de saúde e da adesão ao tratamento e aos cuidados para o controle da doença.

Ao analisar a adesão das mastectomizadas a um programa de reabilitação, Gutiérrez, Bravo, Chanes, De Vivo e Souza (2007) notaram que é necessário promover melhor controle da dor pós-operatória e reforço das orientações para incrementar a adesão ao tratamento do câncer de mama. Ao focalizar o processo adaptativo das mastectomizadas envolvidas em um grupo de apoio, outro estudo concluiu que a participação de entes queridos/familiares na execução do plano de cuidados é essencial para aumentar sua efetividade (Rodrigues, Silva, \& Fernandes, 2003).

\section{CONSIDERAÇÕES FINAIS}

Esta revisão da literatura permitiu sistematizar e analisar resultados de intervenções psicológicas em grupo realizadas com mulheres diagnosticadas com câncer de mama e, nessa perspectiva, oferece aportes que reforçam a relevância desse modelo de intervenção na assistência oncológica, além de permitir uma melhor compreensão dos avanços de pesquisas realizadas na área, bem como a identificação das lacunas existentes e dos aspectos que devem ser abordados em investigações futuras.

O estudo identificou que todos os 28 artigos revisados corroboram alguns dos benefícios proporcionados pelas intervenções de grupo com mulheres mastectomizadas. Os efeitos benéficos do apoio recebido durante a intervenção grupal parecem ser mais marcados naquelas participantes que, inicialmente, apresentavam maior sofrimento psicológico. Isso dá pistas para que estudos futuros se dediquem a investigar detalhadamente os diferentes momentos de realização dos grupos de intervenção, combinando essa variável com o tipo e os estágios do desenvolvimento do grupo, o grau de estadiamento da doença e outros aspectos clínicos e sociodemográficos das participantes.

Entre os ganhos comprovados, encontram-se benefícios de ordem psicológica, como redução do estresse, aumento da qualidade de vida, melhora do humor, compartilhamento de informações sobre os diversos tratamentos, promoção de comportamentos de saúde e habilidades de autocuidado, entre outros, que podem fortalecer a adesão ao tratamento.
Embora em menor quantidade, também foram encontrados estudos que concluíram que os grupos de apoio são de pouco auxílio quanto ao aumento da expectativa de vida dessas mulheres, sugerindo, assim, que sua potencial contribuição permanece restrita à dimensão psicológica, o que, por si só, já é de grande mérito.

Desse modo, os resultados não são conclusivos quanto a responder se intervenções psicológicas em grupo podem melhorar a sobrevida das mulheres acometidas, reduzindo o risco de recidiva e de morte ou contribuindo com outros desfechos secundários, como o reforço do sistema imunológico, considerando que a imunidade fica comprometida em consequência do efeito colateral imunossupressor dos agentes quimioterápicos. Assim, no estágio atual do conhecimento, é possível afirmar que os grupos de apoio às mulheres com câncer de mama conduzem a melhores desfechos psicológicos, porém, as evidências dos efeitos do grupo para o incremento da sobrevida das pacientes não são convincentes (Goodwin, 2005; Goodwin et al., 2001), embora o título do primeiro desses dois artigos sinalize uma expectativa promissora: "grupos de apoio no câncer de mama avançado: viver melhor, se não mais".

Por essa razão, novos estudos devem ser desenvolvidos junto às mulheres que participam de grupos de apoio, sobretudo com recorte longitudinal, para que se possa identificar e validar a estabilidade dos resultados alcançados quanto a possíveis mudanças na qualidade de vida, nas 
condições favoráveis para a recuperação da saúde e na prevenção dos agravos decorrentes do tratamento. Estudos futuros, com delineamentos robustos, poderiam explorar ainda a questão de quais são os tipos e as modalidades de grupo mais efetivas para cada perfil de pacientes e que modelos favorecem a ressignificação das experiências, propiciando, assim, que se tire um legado positivo do trauma vivido.

Em relação às limitações que podem ser apontadas nos artigos revisados, tem-se que na maioria dos estudos foram investigados grupos de pacientes provenientes de um mesmo hospital ou centro de tratamento, o que pode enviesar os resultados. Além disso, nenhum dos estudos foi realizado com mulheres que, por alguma razão, desistiram ou deixaram de participar de intervenções grupais, o que talvez explique porque poucas dificuldades foram relatadas pelas participantes em relação à inserção nos grupos.

Após concluir o exame minucioso da literatura, algumas questões não respondidas até o atual momento pelos estudos e que deveriam ser investigadas no futuro são: o que mantém essas mulheres nos grupos, considerando as necessidades que são atendidas por grupos de apoio específico? Que benefícios são percebidos pelas participantes desses grupos? Como a experiência de sociabilidade em grupo impacta suas vidas e a de seus familiares? Que aprendizagens essas participantes transpõem do grupo para o seu cotidiano? Os benefícios do apoio só se evidenciariam no período em que as mulheres estão participando ativamente da intervenção grupal ou tenderiam a ser generalizados mesmo depois de elas deixarem de frequentar as atividades do grupo, mantendo assim seus efeitos inerciais e/ou duradouros?

\section{REFERÊNCIAS}

Ambrósio, D. C. M., \& Santos, M. A. (2011). Familiares de mulheres com câncer de mama: vivências diante da possibilidade de morte. Psicologia: Teoria e Pesquisa, 27(4), 475-484

Andersen, B. L., Yang, H. C., Farrar, W. B., Golden-Kreutz, D. M., Emery, C. F., Thornton, L. M... Carson III, W. E. (2008). Psychologic intervention improves survival for breast cancer patients. American Cancer Society, 113(12), 3450-3458.

Bandura, A., Pastorelli, C., Barbaranelli, C., \& Caprara, G. V. (1999). Self-efficacy pathways to childhood depression. Journal of Personality and Social Psychology, 76(2), 258-269.

Barbosa, R. C., Ximenes, L. B., \& Pinheiro, A. K. B. (2004). Mulher mastectomizada: Desempenho de papéis e redes sociais de apoio. Acta Paulista de Enfermagem, 17(1), 18-24.

Barrera, I., \& Spiegel, D. (2014) Review of psychotherapeutic interventions on depression in cancer patients and their impact on disease progression. International Review of Psychiatry, 26(1), 31-43.

Beutel, M. E., Weißflog, G., Leuteritz, K., Wiltink, J., Haselbacher, A., Ruckes, C., ... Brähler, E. (2014). Efficacy of short-term psychodynamic psychotherapy (STPP) with depressed breast cancer patients: results of a randomized controlled multicenter trial. Annals of Oncology, 25(2), 378-384.

Björneklett, H. G., Rosenblad, A., Lindemalm, C., Ojutkangas, M. L., Letocha, H., Strang, P., \& Bergkvist, L. (2013). A randomized controlled trial of support group intervention after breast cancer treatment: Results on sick leave, health care utilization and health economy. Acta Oncologica, 52, 38-47.

Boesen, E. H., Karlsen, R., Christensen, J., Paaschburg, B., Nielsen, D., Bloch, I. S ...Johansen, C. (2011). Psychosocial group intervention for patients with primary breast cancer: A randomised trial. European Journal of Cancer, 47(9), 13631372.

Bordeleau, L., Szalai, J. P., Ennis, M., Leszcz, M., Speca, M., Sela, R., . . Goodwin, P. J. (2003). Quality of life in a randomized trial of group psychosocial support in metastatic breast cancer: overall effects of the intervention and an exploration of missing data. Journal of Clinical Oncology, 21(10), 1944-1951.

Brasil. (2017a). Ministério da Saúde. Secretaria de Atenção à Saúde. Instituto Nacional de Câncer José Alencar Gomes da Silva. Coordenação de Prevenção e Vigilância. Tipos de câncer. Rio de Janeiro: INCA. Disponível em $<\mathrm{http}$ ://www2. inca.gov.br/wps/wcm/connect/tiposdecancer/site/home/mama/ cancer mama>.
Brasil (2017b). Ministério da Saúde. Secretaria de Atenção à Saúde. Instituto Nacional de Câncer José Alencar Gomes da Silva. Coordenação de Prevenção e Vigilância. Estimativa 2016: Incidência de câncer no Brasil. Rio de Janeiro: INCA. Disponível em <http://www.inca.gov.br/estimativa/2016/ sintese-de-resultados-comentarios.asp $>$.

Broome, M. E. (2000). Integrative literature reviews in the development of concepts. In B. L. Rodgers \& K. A. Knafl (Eds.), Concept development in nursing: Foundations, techniques and applications (2nd ed., pp. 231-250). Philadelphia, PA: W. B. Saunders.

Caetano, E. A., Gradim, C. V. C., \& Santos, L. E. S. (2009). Câncer de mama: Reações e enfrentamento ao receber o diagnóstico. Revista de Enfermagem UERJ, 17(2), 257-261.

Caetano, E. A., Panobianco, M. S., \& Gradim, C. V. C. (2012). Análise da produção científica nacional sobre a utilização de grupos na reabilitação de mastectomizadas. Revista Eletrônica de Enfermagem, 14(4), 965-973.

Cameron, L. D., Booth, R. J., Schlatter, M., Ziginskas, D., Harman, J. E., \& Benson, S. R. C. (2005). Cognitive and affective determinants of decisions to attend a group psychosocial support program for women with breast cancer. Psychosomatic Medicine, 67(1), 584-589.

Carlson, L. E., Doll, R., Stephen, J., Faris, P., Tamagawa, R., Drysdale, E., \& Speca, M. (2013). Randomized controlled trial of mindfulness-based cancer recovery versus supportive expressive group therapy for distressed survivors of breast cancer. Journal of Clinical Oncology, 31(25), 3119-3126.

Cavalcanti, P. P., Fernandes, A. F. C., \& Rodrigues, M. S. P. (2002). A interação no grupo de auto-ajuda: Suporte na reabilitação de mulheres mastectomizadas. RENE: Revista da Rede de Enfermagem do Nordeste, 3(1), 47-51.

Cesnik, V. M., \& Santos, M. A. (2012a). Mastectomia e sexualidade: Uma revisão integrativa. Psicologia: Reflexão e Crítica, 25(2), 339-349.

Cesnik, V. M., \& Santos, M. A. (2012b). Do the physical discomforts from breast cancer treatments affect the sexuality of women who underwent mastectomy?. Revista da Escola de Enfermagem da USP, 46(4), 1001-1008.

Cesnik, V. M., Vieira, E. M. V., Giami, A., Almeida, A. M., Santos, D. B., \& Santos, M. A. (2013). The sexual life of women with breast cancer: Meanings attributed to the diagnosis and its impact on sexuality. Estudos de Psicologia (Campinas), 30(2), 187-197. 
Classen, C. C., Kraemer, H. C., Blasey, C., Giese-Davis, J., Koopman, C., Palesh, O. G. . . . Spiegel, D. (2008). Supportiveexpressive group therapy for primary breast cancer patients: A randomized prospective multicenter trial. Psycho-Oncology, 17(5), 438-447.

Cohen, M., \& Fried, G. (2007). Comparing relaxation training and cognitive-behavioral group therapy for women with breast cancer. Research on Social Work Practice, 17(3), 313-323.

Dolbeault, S., Cayrou, S., Brédart, A., Viala, A. L., Desclaux, B., Saltel, P. . . Dickes, P. (2009). The effectiveness of a psychoeducational group after early-stage breast cancer treatment: results of a randomized French study. Psycho-Oncology, 18(6), 647-656.

Emslie, C., Whyte, F., Campbell, A., Mutrie, N., Lee, L., Ritchie, D., \& Kearney, N. (2007). 'I wouldn't have been interested in just sitting round a table talking about cancer': Exploring the experiences of women with breast cancer in a group exercise trial. Health Education Research, 22(6), 827-838.

Fernandes, A. F. C., Barbosa, I. C. F., \& Silva, R. M. (2002). Saúde e ambiente terapêutico na reabilitação de mulheres mastectomizadas. Texto \& Contexto Enfermagem, 11(3), 21-26.

Fernandes, A. F. C., Cavalcanti, P. P., Bonfim, I. M., \& Melo, E. M. (2005). Significado do grupo de autoajuda na reabilitação da mulher mastectomizada. REME: Revista Mineira de Enfermagem, 9(1), 47-51.

Fernandes, A. F. C., Rodrigues, M. S. P., \& Cavalcanti, P. P. (2004), Comportamento da mulher mastectomizada frente às atividades grupais. Revista Brasileira de Enfermagem, 57(1), 31-34.

Ferreira, M. L. S. M., \& Mamede, M. V. (2003). Representação do corpo na relação consigo mesma após mastectomia. Revista Latino-Americana de Enfermagem, 11(3), 299-304.

Geiger, A. M., Mullen, E. S., Sloman, P. A., Edgerton, B. W., \& Petitti, D. B. (2000). Evaluation of a breast cancer patient information and support program. Effective Clinical Practice, 3(4), 157-165.

Glaser, S. R., \& Glassman, R. (2014). Group work with individuals with chronic cancer. Social Work in Health Care, 53(1), 31-47.

Goldstein, D., \& Frantsve, L. S. (2009). Group psychotherapy for chronic illness: Clinical applications of selected empirical studies. International Journal of Group Psychotherapy, 59(4), 577-583.

Gomes, F. A., Panobianco, M. S., Ferreira, C. B., Kebbe, L. M., \& Meirelles, M. C. C. C. (2003). Utilização de grupos na reabilitação de mulheres com câncer de mama. Revista Enfermagem UERJ, 11(3), 292-295.

Gonçalves, C. O., Tavares, M. C. G. C. F., Campana, A. N. N. B., Cabello, C., \& Shimo, A. K. K. (2012). Instrumentos para avaliar a imagem corporal de mulheres com câncer de mama. Psicologia: Teoria e Prática, 14(2), 43-55.

Goodwin, P. J. (2005). Support groups in advanced breast cancer: Living better if not longer. Cancer, 104(11), 2596-1601.

Goodwin, P. J., Leszcz, M., Ennis, M., Koopmans, J., Vincent, L., Guther, H. . . . Hunter, J. (2001). The effect of group psychosocial support on survival in metastatic breast cancer. The New England Journal of Medicine, 345(24), 1719-1726.

Gray, R. E., Fitch, M., Davis, C., \& Phillips, C. (2000). Challenges of participatory research: reflections on a study with breast cancer self-help groups. Health Expectations, 3(4), 243-252.

Gutiérrez, M. G. R., Bravo, M. M., Chanes, D. C., De Vivo, C. R., $\&$ Souza, G. O. (2007). Adesão de mulheres mastectomizadas ao início precoce de um programa de reabilitação. Acta Paulista de Enfermagem, 20(3), 249-254.

Hamama-Raz, Y., Perry, S., Pat-Horenczyk, R., Bar-Levav, R., \& Stemmer, S. M. (2012). Factors affecting participation in group intervention in patients after adjuvant treatment for early-study breast cancer. Acta Oncologica, 51(2), 208-214.

Kent, E. E., Alfano, C. M., Smith, A. W., Bernstein, L., McTiernan, A., Baumgartner, K. B., \& Ballard-Barbash, R. (2013). The roles of support seeking and race/ethnicity in posttraumatic growth among breast cancer survivors. Journal of Psychosocial Oncology, 31(4), 1-18.

Kissane, D. W., Grabsch, B., Clarke, D. M., Smith, G. C., Love, A. W., Bloch, S. . . Li, Y. (2007). Supportive-expressive group therapy for women with metastatic breast cancer: survival and psychosocial outcome from a randomized controlled trial. Psycho-Oncology, 16(4), 277-286.

Kleba, M. E. \& Wendausen, A. (2009). Empoderamento: Processo de fortalecimento dos sujeitos nos espaços de participação social e democratização política. Saúde e Sociedade, 18(4), 733-743.

Lazarus, R. S. \& Folkman, S. (1984). Stress, appraisal and coping. New York: Springer Publishing Company.

Leite, L. P., \& Peres, R. S. (2013). Grupos de apoio a mulheres acometidas por câncer de mama. Revista da SPAGESP, 14(1), 55-67.

Machavoine, J.-L. (2010). Groupes de malades en psychooncologie: Éléments historiques, cliniques et pratiques. Psycho-Oncologie, 4(3), 190-198.

Martins, M. M. \& Peres, R. S. (2014). Fatores terapêuticos em grupo de apoio a mulheres com câncer de mama. Psicologia, Saúde e Doenças, 15(2), 396-407.

Mendes, K. D. S., Silveira, R. C. C. P., \& Galvão, C. M. (2008). Revisão integrativa: Método de pesquisa para a incorporação de evidências na saúde e na enfermagem. Texto \& Contexto Enfermagem, 17(4), 758-764.

Minayo, M. C. (2008). O desafio do conhecimento: Pesquisa qualitativa em saúde. São Paulo: Hucitec; Rio de Janeiro: Abrasco.

Mother, D, Liberati, A, Tetzlaff, J., \& Altman, D. G. (2009). The PRISMA Group Preferred Reporting Items for Sistematic Reviews and Meta-Analyses: the PRISMA statement. Journal of Clinical Epidemiology, 62(10), 1006-1012.

Naaman, S., Radwan, K., Fhaergusson, D., \& Johnson, S. (2009). Status of psychological trials in breast cancer patients: A report of three meta-analyses. Psychiatry, 72(1), 50-69.

Namkoong, K., Shah, D. V., Han, J. Y., Kim, S. C., Yoo, W., Fan, D. . . Gustafson, D. H. (2010). Expression and reception of treatment information in breast cancer support groups: how health self-efficacy moderates effects on emotional well-being. Patient Education and Counseling, 81(1), 41-47.

Oliveira, M. S., Souza, A. M. A., \& Fernandes, A. F. C. (2008). Grupo apoyo/soporte: Espacio de rehabilitación para mujeres mastectomizadas. Revista Eletrônica de Enfermagem, 10(3), 816-822.

Palesh, O. G., Collie, K., Batiuchok, D., Tilston, J., Koopman, C., Perlis, M. L., . . Spiegel, D. (2007). A longitudinal study of depression, pain, and stress as predictors of sleep disturbance among women with metastatic breast cancer. Biological Psychology, 75(1), 37-44.

Peres, R. S. \& Santos, M. A. (2007). Breast cancer, poverty and mental health: Emotional response to the disease in women from popular classes. Revista Latino-Americana de Enfermagem, 15(número especial), 786-791.

Peres, R. S. \& Santos, M. A. (2009). Personalidade e câncer de mama: Produção científica em Psico-Oncologia. Psicologia: Teoria e Pesquisa, 25(4), 611-620.

Peres, R. S. \& Santos, M. A. (2012). Psicossomática psicanalítica: Intersecções entre teoria, pesquisa e clínica. Campinas, SP: Alínea.

Pinheiro, C. P. O., Silva, R. M., Mamede, M. V., \& Fernandes, A. F. C. (2008). Participação em grupo de apoio: experiência de mulheres com câncer de mama. Revista Latino-Americana de Enfermagem, 16(4), 733-738.

Pinho, L. S., Campos, A. C. S., Fernandes, A. F. C., \& Lobo, A. S. (2007). Câncer de mama: Da descoberta à recorrência da doença. Revista Eletrônica de Enfermagem, 9(1), 154-165. 
Rizalar, S., Ozbas, A., Akyolcu, N., \& Gungor, B. (2014). Effect of perceived social support on psychosocial adjustment of Turkish patients with breast cancer. Asian Pacific Journal of Cancer Prevention, 15(8), 3429-3434.

Rodrigues, D. P., Silva, R. M., \& Fernandes, A. F. C. (2003). O processo adaptativo de mulheres mastectomizadas: Grupo de apoio. Revista Enfermagem UERJ, 11(1), 64-69.

Rossi, L., \& Santos, M. A. (2003). Repercussões psicológicas do adoecimento e tratamento em mulheres acometidas pelo câncer de mama. Psicologia: Ciência e Profissão, 23(4), 32-41.

Rowland, J. H., Meyerowitz, B. E., Crespi, C. M., Leedham, B., Desmond, K., Belin, T. R., \& Ganz, P. A. (2009). Addressing intimacy and partner communication after breast cancer: A randomized controlled group intervention. Breast Cancer Research and Treatment, 118(1), 99-111.

Santos, D. B., Ford, N. J., Santos, M., \& Vieira, E. M. (2014). Breast cancer and sexuality: The impacts of breast cancer treatment on the sex lives of women in Brazil. Culture, Health \& Sexuality: An International Journal for Research, Intervention and Care, 16(3), 246-257.

Santos, D. B., Santos, M. A., \& Vieira, E. M. (2014). Sexualidade e câncer de mama: Uma revisão sistemática da literatura. Saúde \& Sociedade, 23(4), 1342-1355.

Santos, M. A. \& Pereira-Martins, M. L. P. L. (2016). Estratégias de enfrentamento adotadas por pais de crianças com deficiência intelectual. Ciência \& Saúde Coletiva, 21(10), 3233-3244. https:// dx.doi.org/10.1590/1413-812320152110.14462016

Santos, M. A., Prado, M. A. S., Panobianco, M. S., \& Almeida, A. M. (2011). Grupo de apoio a mulheres mastectomizadas: Cuidando das dimensões subjetivas do adoecer. Revista da SPAGESP, 12(2), 27-33.

Shannonhouse, L., Myers, J., Barden, S., Clarke, P., Weimann, R., Forti, A... Porter, M. (2014). Finding your new normal: Outcomes of a wellness-oriented psychoeducational support group for cancer survivors. The Journal for Specialists in Group Work, 39(1), 3-28.

Simeão, S. F. A. P., Landro, I. C. R., Conti, M. H. S., Gatti, M. A. N., Delgallo, W. D., \& Vitta, A. (2013). Qualidade de vida em grupos de mulheres acometidas de câncer de mama. Ciência \& Saúde Coletiva, 18(3), 779-788.

Silva, G., \& Santos, M. A. (2008). "Será que não vai acabar nunca?”: Perscrutando o universo do pós-tratamento do câncer de mama. Texto \& Contexto Enfermagem, 17(3), 561-568.

Silva, G., \& Santos, M. A. (2010). Stressors in breast cancer posttreatment: A qualitative approach. Revista Latino-Americana de Enfermagem, 18(4), 688-695.
Souza, B. F., Moraes, J. A. M., Inocenti, A., Santos, M. A., Silva, A. E. B. C., \& Miasso, A. I. (2014). Women with breast cancer taking chemotherapy: Depression symptoms and treatment adherence. Revista Latino-Americana de Enfermagem, 22(5), 866-873.

Spiegel, D. (2001). Mind matters: Group therapy and survival in breast cancer. New England Journal of Medicine, 345(24), 1767-1768.

Spiegel, D., Butler, L. D., Giese-Davis, J., Koopman, C., Miller, E., DiMiceli, S. . . Kraemer, H. C. (2007). Effects of supportiveexpressive group therapy on survival of patients with metastatic breast cancer. American Cancer Society, 110(5), 1130-1138.

Stang, I., \& Mittelmark, M. B. (2008). Learning as an empowerment process in breast cancer self-help groups. Journal of Clinical Nursing, 18(14), 2049-2057.

Tedeschi, R. G. \& Calhoun, L. G. (1996). The Posttraumatic Growth Inventory: Measuring the positive legacy of trauma. Journal of Traumatic Stress, 9(3), 455-471.

Vidotti, J. F., Scorsolini-Comin, F., \& Santos, M. A. (2013). Qualidade de vida em sobreviventes de longo prazo ao câncer de mama: Análise da produção científica. Psicologia: Teoria e Prática, 15(3), 49-68.

Vieira, E. M., Santos, D. B., Santos, M. A., \& Giami, A. (2014). Experience of sexuality after breast cancer: A qualitative study with women in rehabilitation. Revista Latino-Americana de Enfermagem, 22(3), 408-414.

Vilhauer, R. P., McClintock, M. K., \& Matthews, A. K. (2010). Online support groups for women with metastatic breast cancer: A feasibility pilot study. Journal of Psychosocial Oncology, 28(5), 560-586.

Vos, P. J., Visser, A. P., Garssen, B., Duivenvoorden, H. J., \& Haes, H. C. J. M. (2006). Effects of delayed psychosocial interventions versus early interventions for women with early stage breast cancer. Patient Education and Counseling, 60(2), 212-219.

Vos, P. J., Visser, A. P., Garssen, B., Duivenvoorden, H. J., \& Haes, H. C. J. M. (2007). Effectiveness of group psychotherapy compared to social support groups in patients with primary, non-metastatic breast cancer. Journal of Psychosocial Oncology, 25(4), 37-60.

Whittemore, R., \& Knafl, K. (2005). The integrative review: Update methodology. Journal of Advanced Nursing, 52(5), 546-553.

Yavuzsen, T., Karadibak, D., Cehreli, R., \& Dirioz, M. (2012). Effect of group therapy on psychological symptoms and quality of life in Turkish patients with breast cancer. Asian Pacific Journal of Cancer Prevention, 13(11), 5593-5597. 